\title{
EFFECT OF WALL INCLINATION ON THE DYNAMIC BEHAVIOUR OF AN OSCILLATING WATER COLUMN SYSTEM
}

\author{
Abdelhamid El Barakaz**1, Abdellatif El Marjani ${ }^{2}$,Hamid Mounir ${ }^{3}$ \\ EMISys Research Team, Engineering 3S Research Center, Turbomachinery Lab. Mohammadia School of Engineers, University \\ Mohammed V in Rabat, B.P. 765 Agdal, Rabat, Morocco
}

\begin{abstract}
The Oscillating Water Column device (OWC) is one of the most used Wave Energy Converters (WECs) for wave energy harvesting. It consists essentially of two parts: the pneumatic chamber made of concrete and the bidirectional turbine linked to a generator group for energy production.

In this study we are interested in the water motion oscillation inside the chamber resulting from the water level perturbation. This process is characterized by its own natural frequency and global damping. The vertical OWC chamber model is limited by the number of parameters defining the natural frequency and the global damping. The objective of this paper is to improve the performances obtained for the vertical OWC by considering an OWC with inclined sidewalls.

For maximum efficiency, the device must operate in the resonance domain where the damping is low and the frequency of incoming waves matches with the natural frequency of the OWC. This will theoretically amplify the pneumatic energy to be converted to a mechanical one in the turbine.
\end{abstract}

\section{NOMENCLATURE}

$B$

$C$

$d$

$H$

$h$

$k$

$L$

$P$

$P_{0}$

$\Delta P$

$Q$

$Q_{m}$

$S$

$T$
Chamber width

Turbine damping coefficient

Chamber submerged length

Elevation of sea waves

Water elevation inside the chamber

Heat capacity ratio

Height of the chamber

Air pressure inside the chamber

Atmospheric pressure

Pressure drop in the turbine

Air flow rate

Air mass flow rate

Chamber base area

Wave period $\alpha$

$\gamma$

$\rho$

$\rho_{0}$

$\rho_{s}$

$\omega_{0}$

\section{INTRODUCTION}

Nowadays, sea wave energy is being considered in many countries as a promising source of renewable energy. Waves are produced by wind action, and they are therefore an indirect form of solar energy and can be seen as concentrated form of solar power. Indeed, solar power mean received by earth is in the order of $100 \mathrm{~W} \cdot \mathrm{m}^{-2}$, which can be converted into waves with a power concentration of over $60 \mathrm{~kW} \cdot \mathrm{m}^{-1}$ [1].Typical values for good offshore locations in average range are between 20 and $70 \mathrm{~kW} / \mathrm{m}$, particularly, in moderate to high latitudes. Seasonal variations are in general considerably larger in the northern than in the southern hemisphere [2].

${ }^{*}$ Corresponding author: ${ }^{1}$ elbarakaz.abdelhamid@gmail.com 
Converting this energy into a more usable one has inspired numerous inventors and more than one thousand patents had been registered since 1980 [2].Various devices have been performed to exploit this kind of energy, such as: power buoy, wave dragon, Pelamis and the oscillating water column (OWC) [3]

The Oscillating Water Column (OWC) is considered as one of the most studied devices for extracting wave's energy. especially for two great reasons: the first one is its simplicity, and the second one refers to the low maintenance costs due to the absence of moving parts in direct contact with water.

Any OWC system consists of a submerged air chamber in the sea, connected to the atmosphere by means of a circular duct where an air turbine is installed. The periodic movement of water inside the chamber creates inhalation and exhalation of air inside the chamber through the duct and introduces a bidirectional flow through the turbine (Fig. 1). Moving parts of the air turbine should rotate in the same direction despite the air flow changes; turbines like Wells turbine and radial or axial impulse turbine match these conditions [4].

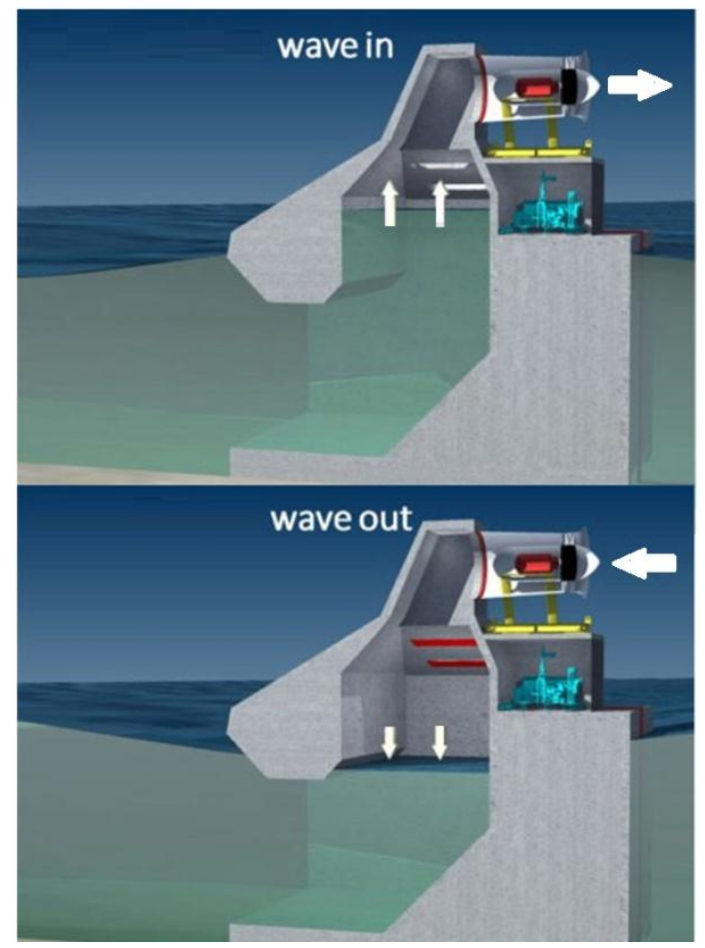

Fig. 1 OWC Working Principle

In the present study, and in order to exploit the resonance phenomenon, a simple one dimensional model has been proposed to investigate the effect of the inclining sidewalls on the OWC performances.

\section{MODELLING}

\subsection{Assumptions:}

Due to the nature of the system, it can be studied in a 2D plan. As depicted in Fig. 2, the height of the water surface inside the chamber is represented by $h$; the chamber has a width $B$ in sea equilibrium level, a height $L$ and a length $d$ for the submerged portion, the left sidewall forms an angle $\alpha$ with the $\mathrm{z}$ axis. The air pressure inside and outside the chamber are respectively $P$ and $P_{0}$, the density of air inside and outside the chamber are $\rho$ and $\rho_{0}$.

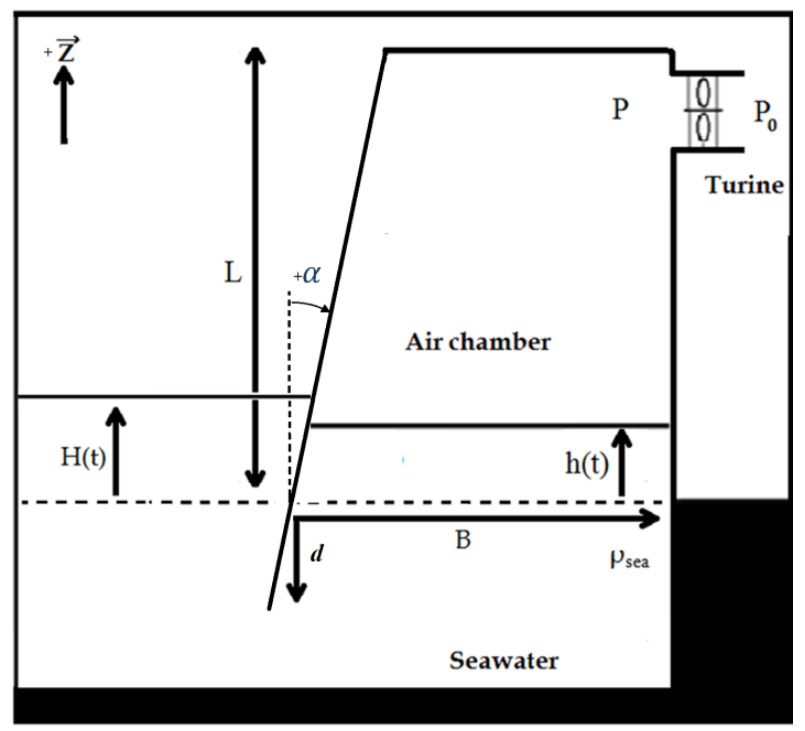

Fig. 2 Geometry of the OWC Chamber

All general assumptions made in the modelling approach are described as follows:

- The chamber free water surface is assumed to move only in the vertical direction.

- The hydraulic energy loss process via viscosity of fluids and frictions is neglected

- The pneumatic energy loss process in the duct and in the air turbine is neglected

- $\quad$ Air is considered as compressible perfect gas, the process of inhalation and exhalation as isentropic transformation.

- Pressure drop inside the turbine has been simulated with a linear law relating the pressure drop to the flow rate (Wells turbine).

- The origin of waves elevation $(\mathrm{h}=0)$ coincides with sea level in equilibrium.

- Z-axis is pointing upward in the vertical direction.

- The cross section $\mathrm{S}$ of the chamber depends only on the inclination and on the height $\mathrm{z}$.

\subsection{The governing equation:}

There are three factors directing the free water surface motion inside the chamber:

- Water hydrodynamics

- Air Chamber aerodynamics

- Turbine effect 
The main equation of each domain leads to understand the physics behind the OWC behaviour and establish the coupled system.

Water equation: Euler equation (equation of the momentum) can be applied on water mass inside the chamber:

$$
\frac{\partial \vec{v}}{\partial t}+\operatorname{grad}\left(\frac{v^{2}}{2}\right)-v \wedge \operatorname{rot} v=-\operatorname{grad} \frac{P}{\rho_{s}}+\boldsymbol{g}
$$

After a scalar multiplication by $\mathbf{d z}$ element parallel to the streamlines: only the scalar field $z$ is taken into account.

$$
\frac{\partial v}{\partial t} \boldsymbol{d z}+d\left(\frac{v^{2}}{2}+\frac{P}{\rho}+g z\right)=0
$$

Then, integration between $-d$ the length of the submerged portion of the chamber and $h$ the water surface elevation inside the chamber to obtain the equation(3):

$$
\int_{-d}^{h} \frac{\partial v}{\partial t} \boldsymbol{d z}+\left[\frac{v^{2}}{2}+\frac{P}{\rho_{s}}+g z\right]_{-d}^{h}=0
$$

The surface of any horizontal section of the chamber depends on $z$ and $\alpha$, and can be expressed as follows:

$$
S(z)=A(B-\tau z) \text { with } \tau=\tan (\alpha)
$$

To calculate water velocity in each section $S(z)$, the continuity condition of flow has been used (for the incompressible water). The flow rate $Q=S . v$ is constant in each section

Hence:

$$
v(z)=\frac{B-\tau h}{B-\tau z} \dot{h}
$$

We follow the reasoning from the equation(3), to obtain the following equation for water domain:

$$
\begin{gathered}
\int_{-d}^{h} \frac{\partial\left(\frac{B-\tau h}{B-\tau z} \dot{h}\right)}{\partial t} d z+\frac{\dot{h}^{2}-\left(\frac{B-\tau h}{B+\tau D} \dot{h}\right)^{2}}{2} \\
+\frac{\Delta P+P_{0}-\left(P_{0}+\rho_{s} g(H+d)\right)}{\rho}+g(h+d)=0
\end{gathered}
$$

After all calculations, the main result for the water domain can be expressed in the following equation:

$$
\begin{array}{r}
{\left[\dot{h}^{2}-\ddot{h}\left(\frac{B}{\tau}-h\right)\right] \ln \left(\frac{B-\tau h}{B+\tau d}\right)} \\
+\dot{h}^{2}\left[1-\left(\frac{B-\tau h}{B+\tau d}\right)^{2}\right]+\frac{\Delta P}{\rho_{s}}=g(H-h)
\end{array}
$$

Air chamber equation: The air inside the chamber had been regarded as a perfect gas and the compression process as an isentropic transformation, the relationship between pressure and the density of air inside the chamber is expressed as follows:

$$
\rho=\rho_{0}\left(\frac{P}{P_{0}}\right)^{\frac{1}{k}}
$$

Then, the mass flow through the duct $Q_{m}$ depends on the mass $\mathrm{m}$ and the volume $\mathrm{V}$ of the enclosed air inside the chamber:

$$
Q_{m}=-\frac{d m}{d t}=-\rho \frac{d V}{d t}-V \frac{d \rho}{d t}
$$

After calculations:

$$
Q_{m}=S \rho(\dot{h}-(L-h)) \frac{1}{k P} \frac{d P}{d t}
$$

Turbine equation: Wells and impulse turbines are the most popular in practical applications. The relation between the pressure drop and the flow rate is linear for Wells turbine and quadratic for impulse turbine [5]. In our case, the linear relation of Wells turbine is adopted:

$$
\Delta P=P-P_{a t m}=C \cdot Q=C \cdot Q_{m} / \rho
$$

Combining equation (9) and(10), we obtain the following expression for the turbine pressure drop:

$$
\Delta P=C . S .\left(\dot{h}-\frac{(L-h)}{k \cdot\left(\Delta P+P_{0}\right)} \frac{d \Delta P}{d t}\right)
$$

Finally the coupled system resulting from equations (6) and(11):

$$
\left\{\begin{array}{c}
{\left[\dot{h}^{2}-\ddot{h}\left(\frac{B}{\tau}-h\right)\right] \ln \left(\frac{B-\tau h}{B+\tau d}\right)} \\
+\dot{h}^{2}\left[1-\left(\frac{B-\tau h}{B+\tau d}\right)^{2}\right]+\frac{\Delta P}{\rho_{s}}=g(H-h) \\
\Delta P=C S\left(\dot{h}-\frac{(L-h)}{k \cdot\left(\Delta P+P_{0}\right)} \frac{d \Delta P}{d t}\right)
\end{array}\right.
$$

\subsection{Numerical Resolution:}

To solve the previous coupled nonlinear system(12), numerical methods are generally the most adopted, the Runge-Kutta method presents good advantages: stable in most cases and used generally for non-stiff system, with a good order of Accuracy.

In our case, Runge-Kutta $4^{\text {th }}$ order method has been applied in the resolution step.

First, we need to rewrite the coupled system (12) as equations of 1 st order. For this, we introduce another variable $U=\dot{h}$ (water vertical velocity). 


$$
\left\{\begin{array}{c}
\dot{h}=U \\
\dot{U}=\frac{g(h-H)}{\left(\frac{B}{\tau}-h\right) \ln \left(\frac{B-\tau h}{B+\tau d}\right)}+\frac{U^{2}\left[1-\left(\frac{B-\tau h}{B+\tau d}\right)^{2}\right]}{\left(\frac{B}{\tau}-h\right) \ln \left(\frac{B-\tau h}{B+\tau d}\right)} \\
+\frac{\Delta P}{\rho_{S}\left(\frac{B}{\tau}-h\right) \ln \left(\frac{B-\tau h}{B+\tau d}\right)}+\frac{U^{2}}{\left(\frac{B}{\tau}-h\right)} \\
\quad \dot{P}=\left(-U+\frac{\Delta P}{C \cdot S}\right) \frac{k\left(\Delta P+P_{0}\right)}{(h-L)}
\end{array}\right.
$$

The following numerical values listed in Table 1 and Table 2 are adopted for the system parameters and initial conditions.

\begin{tabular}{|c|c|}
\hline $\mathrm{g}$ & $9.81 \mathrm{~m} / \mathrm{s}$ \\
\hline $\mathrm{H}$ & $0 \mathrm{~m}$ \\
\hline $\mathrm{k}$ & 1.4 \\
\hline $\mathrm{P}_{0}$ & $10^{5} \mathrm{~Pa}$ \\
\hline$\rho_{\mathrm{s}}$ & $1000 \mathrm{Kg} / \mathrm{m}^{3}$ \\
\hline $\mathrm{L}$ & $5 \mathrm{~m}$ \\
\hline $\mathrm{d}$ & $2 \mathrm{~m}$ \\
\hline B & $10 \mathrm{~m}$ \\
\hline $\mathrm{S}$ & $50 \mathrm{~m}$ \\
\hline $\mathrm{C}$ & $50 \mathrm{~Pa} \cdot \mathrm{m}^{-3} \cdot \mathrm{s}$ \\
\hline$\alpha$ & $30^{\circ}$ \\
\hline \multicolumn{2}{|c|}{ Table 2 Initial Conditions } \\
\hline$h(t=0)$ & $1 \mathrm{~m}$ \\
\hline$\dot{h}(t=0)$ & $0 \mathrm{~m} / \mathrm{s}$ \\
\hline$\Delta P(t=0)$ & $\mathrm{OPa}$ \\
\hline
\end{tabular}

For the simulation, we are interested only on the OWC behaviour after the perturbation of water level inside the chamber: waves outside the chamber are cancelled $(H(t)=0)$ and the OWC system is perturbed by raising the water level inside the chamber $h$ to its maximum level $\left(h_{\max }=1 \mathrm{~m}\right)$, as a consequence it will oscillate according to its natural frequency like any oscillatory system.
The coupled system is solved using Runge-Kutta $4^{\text {th }}$ order with a simulation time step $\Delta \mathrm{t}$ equal to $0.001 \mathrm{~s}$ for a time domain of 20 seconds, the absolute error is fixed at $10^{-5}$. As shown in Fig. 3, The graph, as expected, describes oscillations with a constant frequency of $0.36 \mathrm{~Hz}$, the amplitude of wave elevation inside the chamber decays over time until it equals to zero, which is similar to the case of the under-damped oscillatory linear systems [6].

\section{RESULTS AND DISCUSSION}

\subsection{Comparison with the linear model:}

The linear model of a vertical OWC model has been elaborated in a previous article [7], it gives a good approximation of the free water surface motion inside the chamber, and it is similar to a second order oscillator with a natural frequency $\omega_{0}$ and a damping $\gamma$, the motion equation of water inside the chamber can be formulated as:

With:

$$
\ddot{h}+\gamma \cdot \dot{h}+\omega_{0}^{2} \cdot h=0
$$

$$
\left\{\begin{array}{l}
\omega_{0}=\sqrt{\frac{g}{d}} \\
\gamma=\frac{C . S}{\rho_{s} \cdot d}
\end{array}\right.
$$

As noticed from the previous equation, the natural frequency of the vertical OWC can only be controlled by fixing the length of the submerged portion of the chamber $d$, which is physically limited by the available sea depth under the chamber. This may limit the solutions during the design stage, where it is recommended to size the chamber under the resonance conditions: the natural frequency of the chamber need to match with the frequency of incoming waves and to operate in low damping.

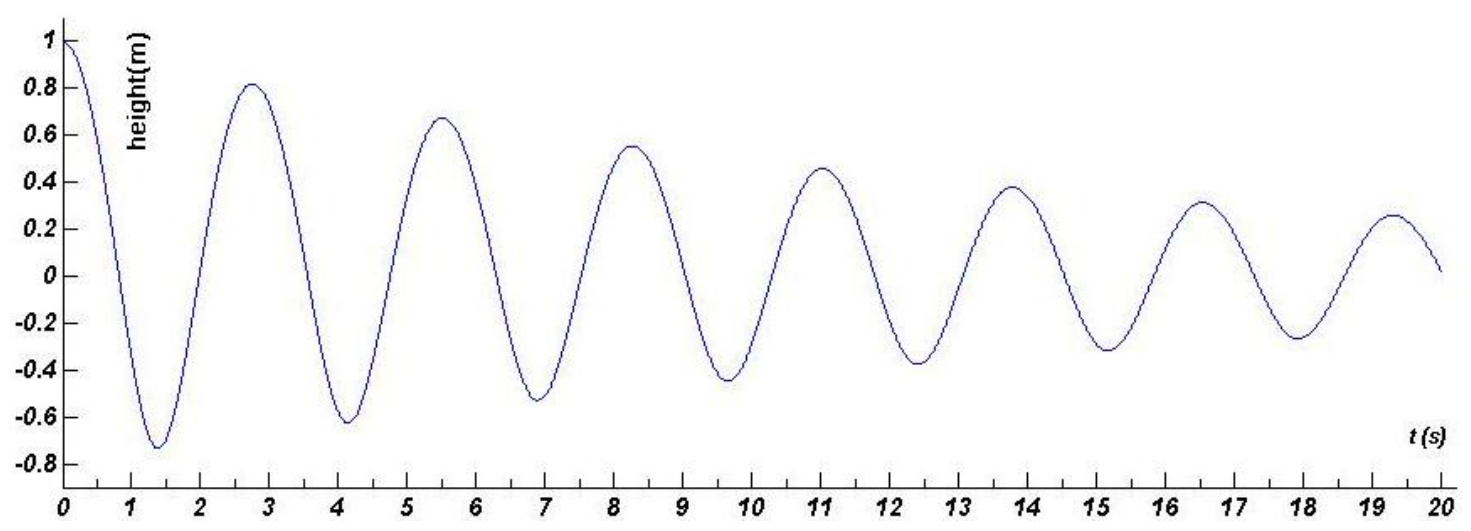

Fig. 3 Runge-Kutta Simulation Solution of the Water Motion 


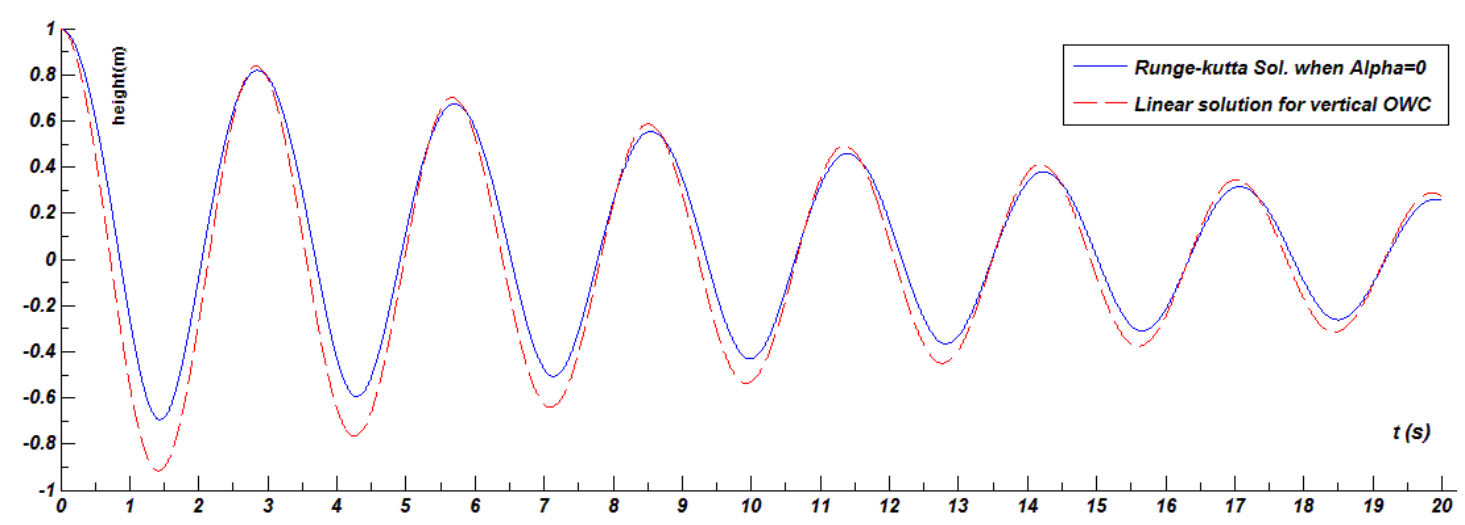

Fig. 4 Comparison between Runge-Kutta Solution $(\alpha=0)$ and the Linear Solution for Vertical OWC

To test the result pertinence of the previous inclined model, it can be compared to the linear model in the same conditions described in Table 1 and Table 2 by taking $\alpha=0$. This changes the inclined OWC to a normal vertical OWC.

From Fig. 4 above, both curves are approximately similar: the constant period of each variation and the amplitude decay are almost the same in both approaches. Despite Certain differences, especially, around the minimum values, this fact is related to the nonlinearity of the coupled system in Runge-Kutta method [8]. It can also be noticed that the aerodynamics inside the chamber (isentropic transformation of the perfect gas, air) have insignificant effect on general results, since the height of the chamber $L$ and the heat capacity ratio $k$ appear in the air equations of the inclined model and have no role in the expressions of linear model. This observation has been confirmed for other scenarios by changing parameters values of Table 1 [9]

\subsection{The effect of inclining sidewalls [10]:}

\subsubsection{OWC Natural frequency:}

As shown in Fig. 5 and Table 3 the angle of inclination affects clearly the natural frequency of the OWC, the period decreased from $3.3 \mathrm{~s}$ to $2.6 \mathrm{~s}$ by changing the value of angle $\alpha$ from $-60^{\circ}$ to $60^{\circ}$, which means a variation of

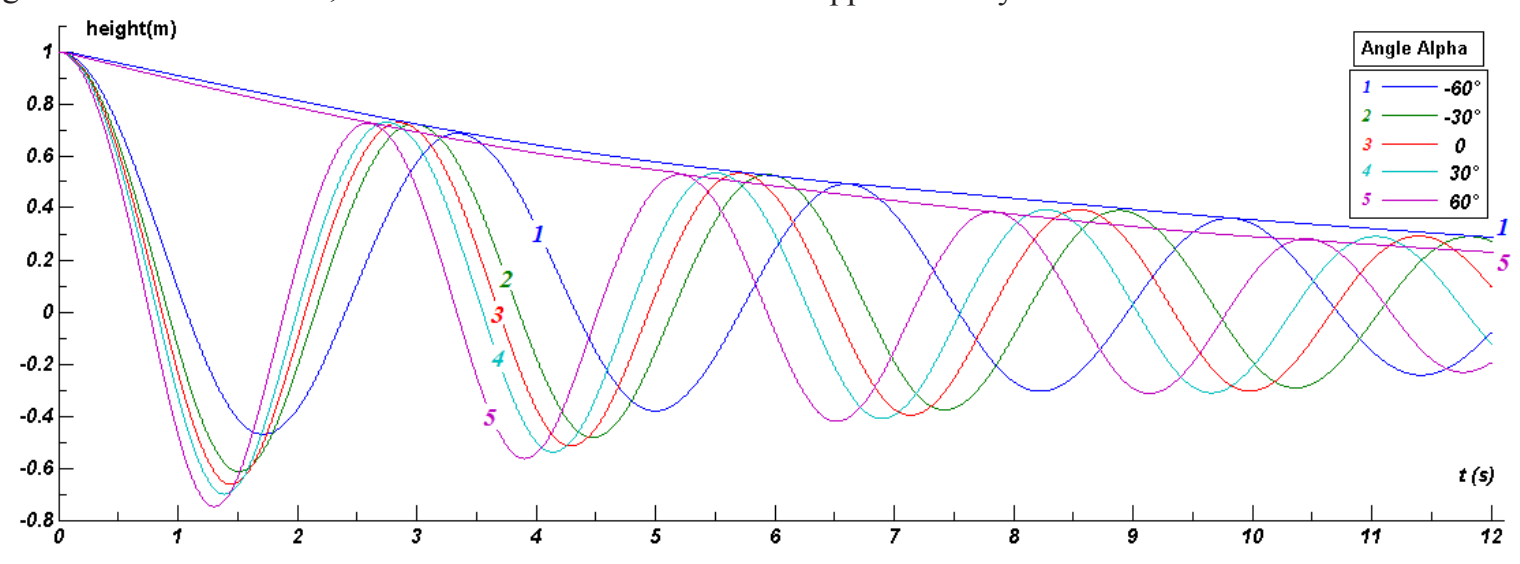

Fig. 5 Effect of Sidewall Angle on Water Motion inside the Chamber natural frequency $\omega_{0}$ proportional to the angle $\alpha$. Values listed in Table 3 show that the effect of inclination can only be observed in large angles, for small angles $\left(|\alpha|<20^{\circ}\right)$ the value of the $\omega_{0}$ is approximately the same (2.1 $\mathrm{rad} / \mathrm{s}=$ vertical OWC freq.)

The proposed model shows that the inclination has minor effect on the natural period: a variation of $120^{\circ}$ from $-60^{\circ}$ to $60^{\circ}$ have changed the natural period of the OWC only by $0.7 \mathrm{~s}$. However, in purpose to operate in the resonance domain, any small amelioration can increase the OWC performances dramatically.

\subsubsection{OWC damping:}

As remarked from both Fig. 5 and Table 3, the global damping variation is proportional to the angle $\alpha$ as the damping becomes stronger when waves are rapidly attenuated. However, the proposed model shows that $\alpha$ has a minor influence on the global damping of the OWC system: the blue cover of the graph corresponding to $-60^{\circ}$ and the purple cover of the graph corresponding to $60^{\circ}$ are close to each other. The difference between the two curves after $10 \mathrm{sec}$ shows an amplitude decay difference of only $7.4 \%$.

Besides, like the natural frequency the effect of inclination can be observed only in large angles, for small angles $\left(|\alpha|<20^{\circ}\right)$ the value of the amplitude decay is approximately $33 \%$. 
Table 3 Inclination Effect

\begin{tabular}{ccc}
\hline Angle $\alpha$ & Freq. $\omega_{0}$ & $\begin{array}{c}\text { Amp. decay } \\
\text { after 10sec }\end{array}$ \\
\hline$-60^{\circ}$ & $1.92 \mathrm{rad} / \mathrm{s}$ & $36.84 \%$ \\
\hline$-40^{\circ}$ & $2.05 \mathrm{rad} / \mathrm{s}$ & $34.69 \%$ \\
\hline$-20^{\circ}$ & $2.16 \mathrm{rad} / \mathrm{s}$ & $33.62 \%$ \\
\hline $0^{\circ}$ & $2.20 \mathrm{rad} / \mathrm{s}$ & $32.95 \%$ \\
\hline $20^{\circ}$ & $2.22 \mathrm{rad} / \mathrm{s}$ & $32.28 \%$ \\
\hline $40^{\circ}$ & $2.32 \mathrm{rad} / \mathrm{s}$ & $31.39 \%$ \\
\hline $60^{\circ}$ & $2.44 \mathrm{rad} / \mathrm{s}$ & $29.47 \%$ \\
\hline
\end{tabular}

\section{CONCLUSIONS}

This paper proposed a method to investigate the effect of inclined sidewalls on the OWC performances, by considering a simple one-dimensional model based on the concept of the water mass oscillations. The OWC study has been divided into three domains to establish the coupled system: Euler equation has been applied for water inside the chamber, the isentropic transformation for the air enclosed inside chamber and the linear Wells law for the air turbine. The resulting nonlinear coupled system has been solved using Runge-Kutta method. Analyses were focused on the temporal variation of water height after perturbing the water level inside the chamber.

Results from the linear vertical OWC and from the studied model by taking a vertical angle are similar. By changing the angle parameter, it has been noticed that the inclination has affected the natural frequency and the global damping of the OWC. As an objective: finding the natural frequency and the global damping then operating with the resonance domain of the OWC device, will theoretically amplify the incoming wave amplitude inside the chamber and improves the efficiency of the OWC, the resonance can be established when the natural frequency, which depends only on the depth $d$ for the vertical OWC, matches with the frequency of incoming sea waves. Considering the inclined sidewalls during the design stage may provide some flexibility and guide for better solutions in the optimisation process.

\section{ACKNOWLEDGMENTS}

This research work has been conducted as part of the research activity within the EMISys research team at the Turbomachinery Lab with the institutions financial support of Mohammadia School of Engineers and Mohammed V University in Rabat.

\section{REFERENCES}

[1] L. Rusu and F. Onea, "The performance of some stateof-the-art wave energy converters in locations with the worldwide highest wave power," Renewable and
Sustainable Energy Reviews, vol. 75, pp. 1348-1362, (2017).

[2] A. F. de O. Falcão, "Wave energy utilization: A review of the technologies," Renewable and Sustainable Energy Reviews, vol. 14, no. 3, pp. 899-918, (2010).

[3] I. López, J. Andreu, S. Ceballos, I.M. de Alegría, and I. Kortabarria, "Review of wave energy technologies and the necessary power-equipment," Renewable and Sustainable Energy Reviews, vol. 27, pp. 413-434, (2013).

[4] M. Takao and T. Setoguchi, "Air Turbines for Wave Energy Conversion," International Journal of Rotating Machinery, vol. 2012, p. 10, (2012).

[5] W. Sheng, T. Lewis, and R. Alcor, "On wave energy extraction of oscillating water column device," in The 4th International Conference on Ocean Energy (ICOE), Dublin, Ireland, (2012).

[6] D. Morin. Waves. [Online]. retrieved from: http://www.people.fas.harvard.edu/ djmorin/book.html

[7] A. El Barakaz and A. El Marjani, "The oscillatory free water surface motion inside OWC chamber for wave energy conversion," in The 4th International Renewable and Sustainable Energy Conference (IRSEC'16) , Marrakech - Ouarzazate, Morocco, (2016)

[8] S. J. Ashlin, S.A. Sannasiraj, and V. Sundar, "Wave Forces on an Oscillating Water Column Device," Procedia Engineering, vol. 116, pp. 1019-1026, (2015).

[9] A. El Barakaz and A. El Marjani, "Analysis of aerohydrodynamic equations inside an OWC device for wave energy conversion," in The 5th International Renewable and Sustainable Energy Conference (IRSEC'17), Tangier, Morocco, (2017).

[10] M. Iino, T. Miyazaki, H. Segawa, and M. Iida, "Effect of inclination on oscillation characteristics of an oscillating water column wave energy converter," Ocean Engineering, vol. 116, pp. 226-235, (2016). 